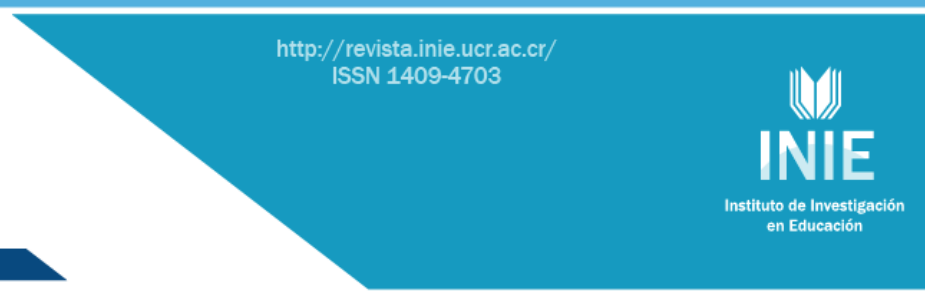

\title{
VALIDACIÓN DE CONTENIDO LÉXICO DE LOS TEXTOS PARA LA COMPRENSIÓN LECTORA DEL TEST DE LECTURA Y ESCRITURA EN ESPAÑOL (LEE) PARA SU APLICACIÓN EN COSTA RICA CONTENT VALIDATION LEXIS OF TEXTS FOR READING COMPREHENSIÓN TEST OF READING AND WRITING SPANISH (LEE) FOR APPLICATION IN COSTA RICA
}

\section{Volumen 16, Número 2} Mayo-Agosto

pp. 1-28

María de los Ángeles Carpio Brenes

Ericka Méndez Chacón

Revista indizada en REDALYC, $\underline{\text { SCIELO }}$

Revista distribuida en las bases de datos:

LATINDEX, DOAJ, E-REVIST@S, IRESIE, CLASE, DIALNET, SHERPA/ROMEO, QUALIS,

Revista registrada en los directorios:

ULRICH'S, REDIE, RINACE, OEI, MAESTROTECA, PREAL, CLACSO 


\title{
VALIDACIÓN DE CONTENIDO LÉXICO DE LOS TEXTOS PARA LA COMPRENSIÓN LECTORA DEL TEST DE LECTURA Y ESCRITURA EN ESPAÑOL (LEE) PARA SU APLICACIÓN EN COSTA RICA \\ CONTENT VALIDATION LEXIS OF TEXTS FOR READING COMPREHENSIÓN TEST OF READING AND WRITING SPANISH (LEE) FOR APPLICATION IN COSTA RICA
}

\author{
María de los Ángeles Carpio Brenes ${ }^{1}$ \\ Ericka Méndez Chacón ${ }^{2}$
}

\begin{abstract}
Resumen: Este artículo presenta los resultados de la validación de contenido léxico de seis textos para la comprensión lectora del Test de Lectura y Escritura en Español (LEE), que se realizó en el II Ciclo 2013 como primer paso para la adaptación de ese instrumento a nuestro contexto educativo costarricense, pues al ser una batería de pruebas argentino-española, el vocabulario se ajusta al utilizado en Costa Rica. Para sustentar esta afirmación, se hizo este estudio con un enfoque instrumental utilizando metodología cuantitativa, donde se utilizó la triangulación para el análisis de los datos suministrados por tres fuentes de información: estudiantes, docentes y personas expertas. Los resultados demuestran que el léxico de los textos es comprensible para una muestra de 477 estudiantes de segundo, tercero y cuarto grados de 15 escuelas del Circuito 09 de la Región Educativa de Cartago; estos datos están apoyados por el criterio de las 35 personas docentes y 5 expertas participantes. Se identificaron muy pocas palabras en los seis textos que podrían generar confusión en la comprensión de los mismos por un asunto de léxico (términos propios de cada región), de las cuales se recomendarán sinónimos como ajuste del test para la segunda parte de este estudio, que corresponde a la determinación de la confiabilidad y validez de contenido, de constructo y de criterio de la totalidad del test para su uso en Costa Rica.
\end{abstract}

Palabras clave: APRENDIZAJE, EDUCACIÓN, EVALUACIÓN, LECTURA, ESCRITURA, COMPRENSIÓN.

\begin{abstract}
This article shows the results of a content validation lexis of six texts for the reading comprehension of the Reading and Writing Test in Spanish (LEE for its acronym in Spanish), as a first step to adapt this instrument to our Costa Rican educational context. Since it is an Argentinian-Spanish set of tests, its vocabulary adjusts easily to the one used in Costa Rica. To support this affirmation, this study was done with an instrument approach employing a quantitative methodology that uses triangulation to analyze given data from three different information sources: students, teachers, and experts. The results show that the vocabulary in the texts is understandable to a group of 477 students from second, third, and fourth grade in 15 schools of the 09 Circuit in the Educational Region of Cartago; these data are supported by the criteria of 35 teachers and 5 experts who participated. Very few words were identified within the six texts that could create confusion due to a vocabulary issue (regional terms); thus, synonyms would be recommended to adjust the test for the second part of this study, which corresponds to the verification of the validity and the reliability of the test to be used in Costa Rica.
\end{abstract}

Key words: LEARNING, EDUCATION, EVALUATION, READING, WRITING, COMPREHENSION

\footnotetext{
1 Docente de la Escuela de Orientación y Educación Especial, Universidad de Costa Rica. Master en Psicopedagogía. Dirección electrónica: maria.carpiobrenes@ucr.ac.cr

2 Docente de la Escuela de Estadística, Universidad de Costa Rica. Máster en Estadística. Dirección electrónica: ericka.mendez@ucr.ac.cr
}

Artículo recibido: 22 de agosto, 2015

Enviado a corrección: 19 de octubre, 2015

Aprobado: 22 de febrero, 2016 


\section{Introducción}

La validación de contenido léxico del Test de Lectura y Escritura en Español (LEE) para su aplicación en estudiantes de Costa Rica resulta importante para el ámbito educativo, porque en esta disciplina no se cuenta con ninguna prueba estandarizada de evaluación diagnóstica de la lectura que esté adaptada a nuestro país. Además, es útil por las características de este instrumento y las áreas que evalúa (conciencia fonológica, lectura y comprensión), resulta pertinente y oportuno para nuestro contexto escolar, pues encaja con el enfoque fonológico para la enseñanza de la lectoescritura inicial que promueve el nuevo Programa de Español del Ministerio de Educación Pública (Costa Rica, Ministerio de Educación Pública, 2014).

La única experiencia como sistema de medición formal planteada en Costa Rica se desarrolló entre 1986 a 1988 como una iniciativa del Instituto de Investigación para el Mejoramiento de la Educación Costarricense (IIMEC) de la Universidad de Costa Rica, cuando se elaboraron pruebas nacionales con referencia a criterios con carácter diagnóstico, censual, en $3^{\circ}, 6^{\circ}, 9^{\circ}$ y $11^{\circ}$ grados, que incluía no solo Español, sino Ciencias, Matemáticas y Estudios Sociales. En 1988 las pruebas se administraron a una muestra y se incluyeron Inglés y Francés en $9^{\circ}$ grado. A partir de 1988 adquirieron normas con carácter sumativo para $11^{\circ}$ grado, llamadas pruebas de Bachillerato; y para $6^{\circ}$ grado en 1989. En 1993 se agregaron para las pruebas sumativas una muestra de $3^{\circ}$ y $9^{\circ}$ grados. En la actualidad, solamente la prueba de Bachillerato para $11^{\circ}$ grado se mantiene.

Posteriormente, en el año 2009 Costa Rica, junto con otros siete países, ingresó al programa de Evaluación Internacional de Alumnos (PISA, por sus siglas en inglés) promovido por la Organización para la Cooperación y el desarrollo Económico (OCDE). Este es un estudio comparativo, internacional y periódico que evalúa el rendimiento del estudiantado de 15 años, al finalizar la etapa escolar obligatoria, a partir de la evaluación de ciertas competencias consideradas claves, como la lectora, la matemática y la científica. Este grupo de países administró en el 2010 los mismos instrumentos utilizados en PISA 2009. La prueba se aplicó en junio del 2010, con la participación de 4565 estudiantes de 181 instituciones de secundaria de todo el país, tanto diurnos, como técnicos y nocturnos, urbanos y rurales, públicos y privados para una cobertura general de todos los segmentos 0 estratos que conformaban la educación secundaria del país (Costa Rica, Ministerio de Educación Pública, 2012). 
PISA evalúa la comprensión de lectura con una escala de seis niveles desde una complejidad mínima -como identificar información puntual en un texto- hasta el nivel 6 que exige la capacidad de crear hipótesis y evaluar con sentido crítico. Según los resultados obtenidos por Costa Rica, nuestra juventud posee al menos un dominio básico de lectura (nivel 2), pero tiene dificultades para responder preguntas que requieren mayor análisis e interpretación. En el área de lectura, alcanzamos el segundo lugar entre 10 países de América Latina que participaron en PISA, solo Chile recibió un puntaje mayor (449) que Costa Rica (443). La gran mayoría de nuestro estudiantado (67\%) mostró el dominio de lectura básico, comparado con 53\% de personas de Latinoamérica.

Costa Rica alcanzó en ese momento la posición 44 entre 74 países, quedando en la mitad inferior de la clase en comprensión de lectura. Mientras el 57\% del estudiantado en los países de la OCDE pudo interpretar textos que requieren una comprensión más que "básica" (nivel 3), solo 33\% del nuestro lo logró. Estos resultados contrastaron aún más con los de países líderes en educación, como Finlandia, donde el 75\% se ubicó en el nivel 3 o por encima de él.

Lamentablemente, la situación no ha mejorado, pues el TERCE: Tercer Estudio Regional Comparativo y Explicativo de la Organización de las Naciones Unidas para la Educación, la Ciencia y la Cultura (UNESCO, 2013) reporta que, a pesar de mantener el segundo lugar de la región con los mejores resultados, las estudiantes y los estudiantes costarricenses empeoraron su nivel de comprensión de lectura durante el período de ocho años, al comparar los datos obtenidos en el SERSE: Segundo Estudio Regional Comparativo y Explicativo (UNESCO, 2008).

TERCE es un estudio a gran escala, aplicado en el 2013 en Costa Rica y otros 14 países (Argentina, Brasil, Chile, Colombia, Ecuador, Guatemala, Honduras, México, Nicaragua, Panamá, Paraguay, Perú, República Dominicana y Uruguay), que evalúa el desempeño de estudiantes de tercer y sexto grado de escuela primaria en las áreas de Matemática, Lectura y Escritura (Lenguaje), y Ciencias Naturales en el caso de sexto grado. Su objetivo principal es dar cuenta de la calidad de la educación en la región y guiar la toma de decisiones en políticas públicas educativas.

La iniciativa de validar el Test de Lectura y Escritura en Español: LEE (Defior, Fonseca, Gotheil, Aldrey, Jiménez, Pujals, Rosa y Dolores, 2006) para su posible uso en nuestro país, se originó porque la investigadora principal lo utilizó en los años 2010 y 2011 como prueba para determinar la competencia lectora de 333 estudiantes costarricenses que conformaron 
la muestra de su investigación para optar por el grado de Doctora por la Universidad Autónoma de Madrid. El instrumento fue recomendado por el profesor tutor, porque se ajustaba a las demandas del estudio y a las características léxicas de nuestra población, pues fue diseñada por un grupo de investigación argentino-español. Durante la aplicación de las pruebas a esta muestra, se pudo observar que el alumnado no mostró dificultad con el vocabulario ni con la estructura de las oraciones planteadas, por lo que se consideró apropiada para el resto del estudiantado costarricense.

En el 2013 se contactó con la Dra. Sylvia Defior, autora del Test LEE y una de las asesoras del nuevo Programa de Español del MEP, quien indicó que no se debía corregir la prueba original que distribuye la Editorial PAIDÓS, sino que debía hacerse el instructivo con las adaptaciones léxico-sintácticas y los baremos correspondientes al contexto costarricense, para su posterior incorporación en el paquete de pruebas del test. Esto implica entonces tres investigaciones: la de validación del contenido, la de confiabilidad del instrumento y la de determinación de los baremos para Costa Rica; este artículo corresponde a los resultados de la validación de contenido léxico de los seis textos que se proponen para la evaluación de la comprensión lectora en el Test LEE.

\section{Referente teórico}

La lectura implica desde reconocer los patrones gráficos del texto hasta la extracción de la intensión comunicativa de quien lo escribe. Es una habilidad que requiere pasar primero por procesos básicos para la decodificación lectora, como lo son la conciencia fonológica, las reglas de correspondencia grafema fonema y el procesamiento léxico, hasta llegar a procesos más complejos, entre los que se destacan el procesamiento sintáctico y el procesamiento semántico (Cuetos, 2008).

La decodificación lectora se refiere a "la capacidad de traducir o descifrar los símbolos escritos al lenguaje oral que el niño ya posee" (De Klerk y Simons, 1989, p. 20). En el caso del idioma español, su sistema de símbolos o código ortográfico es alfabético, lo que quiere decir que el grafema, letra o secuencia de letras, representa un fonema, un sonido específico del idioma. La adquisición de este código resulta fundamental para la persona lectora principiante, ya que le da la clave para realizar una lectura autónoma al permitirle identificar palabras nuevas o poco familiares usando, como mecanismo para la decodificación, las reglas de correspondencia grafema fonema (RCGF). Desde el enfoque psicolingüístico actual, resulta indispensable desarrollar las destrezas psicológicas y del lenguaje que le 
permitan al estudiantado decodificar, tanto desde la ruta léxica, para reconocer la representación gráfica cuando se trata de una palabra conocida, como desde la ruta fonológica, para identificar las palabras mediante el desarrollo de la conciencia fonológica (Sans, 2008).

De acuerdo con Lozano y Lozano (1999), la conciencia fonológica es "tener habilidades para reflexionar conscientemente sobre los diferentes segmentos fonológicos del lenguaje oral" (p. 137). En otras palabras, es una habilidad metalingüística del lector/a principiante (Seymour, 2007), porque le da la capacidad para tratar el lenguaje objetivamente, para reflexionar sobre él de forma explícita y manipular sus estructuras fuera de su función comunicativa (Defior y Serrano, 2011). Lo metalingüístico se convierte, según la lingüística, en un nuevo nivel de actividad del hablante, en el que no sólo el lenguaje se usa durante situaciones comunicativas, sino que, además, la persona lo aísla del contexto inmediato para reflexionar, analizar y examinar sus diferentes dimensiones (Romero, Torrado y Mesa, 2006).

Para algunos autores como Huerta y Matamala (1995), las dimensiones del lenguaje oral se pueden segmentar mediante tres operaciones: la conciencia léxica, que es reflexionar sobre las palabras que forman la frase u oración; la conciencia silábica, que implica segmentar cada palabra en sus sílabas correspondientes; y la conciencia fonémica, que incluye el dividir las palabras en fonemas. Otras autoras, como Herrera y Defior (2005) dejan por fuera el nivel de conocimiento léxico y se refieren a los niveles silábico, fonémico e intrasilábico, que es la habilidad de manipular el ataque (constituido por la consonante o bloque de consonantes iniciales) y la rima (formada por la vocal y las consonantes siguientes). En un estudio reciente de Defior y Serrano (2011), se concilian ambas posiciones al definirse la conciencia fonológica como el conocimiento de que el habla puede dividirse en unidades que incluyen cuatro niveles distintos: la conciencia léxica, la conciencia silábica, la conciencia intrasilábica y la conciencia fonémica.

Sin embargo, estas autoras afirman que de los cuatro niveles, la conciencia fonémica es el mejor predictor de los logros en lenguaje escrito y es uno de los pilares para su aprendizaje, pues de él depende la adquisición de las reglas de correspondencia grafema fonema (RCGF). Phillips y Torgesen (2006) sostienen que contribuye al desarrollo de la precisión de la lectura, principalmente a través de su impacto en el nivel de desarrollo de habilidades de decodificación fonológica, entendida como la capacidad de utilizar el conocimiento de las relaciones entre letras y sonidos junto con la habilidad de fusión 
fonológica al pronunciar palabras desconocidas. Por su parte, McGuinness (1999) afirma que de todas las destrezas medidas por las pruebas, las de análisis fonémico resultan ser, precisamente, las más predictivas de la habilidad lectora.

Según Silva (2015), la conciencia fonológica y el conocimiento de las reglas de correspondencia grafema fonema "constituyen los dos mejores predictores de un buen desarrollo lector durante los dos primeros años de enseñanza” (p. 8), y constituyen los elementos del primer nivel de procesamiento del lenguaje que converge en la comprensión lectora, el nivel perceptivo-fonológico. El segundo nivel para esta autora es el nivel léxico, que implica el acceso a los aspectos superficiales del mensaje mediante el reconocimiento de las palabras y la identificación de sus significados en el diccionario mental. Para Cuetos (2008), el procesamiento léxico es la capacidad de acceder, en forma automática, al significado y la fonología de una palabra.

Según el autor, la persona lectora experimentada ha leído tantas veces las palabras que en cuando aparece alguna en su campo visual no puede dejar de leerla ni de activar su significado y pronunciación. Esto se debe principalmente a las características de las palabras, pues suelen reconocerse más fácilmente aquellas que son reales (lexicalidad), las que son de alta frecuencia, las que se adquieren en edad temprana, las que se ajustan a las reglas de pronunciación grafema-fonema (regularidad), las que son imaginables, las palabras cortas y las que poseen vecindad ortográfica, es decir, que sólo se diferencian en una letra.

Este procesamiento léxico es fundamental para la comprensión lectora, pero insuficiente, ya que las palabras aisladas no transmiten ninguna información nueva sino que es en la relación entre ellas donde se encuentra el mensaje. El objetivo de la lectura es "comprender el contenido del texto, esto es, saber de qué habla el autor, qué nos dice de aquello de lo que nos habla y con qué intención o propósito lo dice" (Tapia, 2005). Para lograrlo, Cuetos (2008) y Silva (2015) coinciden en que se requieren otras dos operaciones o niveles cognitivos, más complejos, de orden superior: el nivel semántico y el nivel sintáctico.

El tercer nivel, el sintáctico, se centra en segmentar cada oración en sus componentes, clasificar cada uno de estos de acuerdo con sus papeles gramaticales y, finalmente, construir una estructura o marco sintáctico que haga posible la extracción del significado. Esta competencia permite captar las relaciones sintagmáticas dentro de un enunciado, así como las relaciones que se producen en el interior de un texto, mediante tres operaciones principales: la asignación de las etiquetas correspondientes a los distintos grupos de palabras que componen la oración (sustantivo, adjetivo, verbo, frase subordinada, etc.); 
especificación de las relaciones existentes entre estos componentes; y la construcción de la estructura correspondiente, mediante ordenamiento jerárquico de los componentes (García, 1993).

Núñez (2009) explica el nivel sintáctico como la manifestación en la capacidad de utilizar el orden de palabras y la concordancia para reconocer las relaciones funcionales en el interior del enunciado; remitir correctamente las informaciones identificando diferentes elementos gramaticales y contextuales; y reconstruir mentalmente la estructura sintáctica lineal a partir de un texto. Según el autor, la competencia sintáctica en el lenguaje escrito interviene en mayor grado que en el oral, porque en este último la entonación, el acento, el tono -y otros elementos- proporcionan también significado; es algo que no ocurre en el escrito, solo las relaciones sintácticas permiten este conocimiento.

García (1993) señala que el reconocimiento de palabras (procesamiento léxico) y las relaciones entre ellas (procesamiento sintáctico) posibilitan extraer su significado, que corresponde al cuarto nivel de procesamiento de lenguaje que converge en la comprensión lectora: el semántico. Según este autor, el procesamiento semántico es "saber captar la relación entre los significantes y los significados aplicando la propia experiencia y los modelos conceptuales adquiridos" (García, 1993, p. 12). Se trata de construir una representación mental del contenido del texto y de integrar esa representación en los propios conocimientos, pues solo en ese caso se produce la auténtica comprensión (Cuetos, 2008). Esta representación no consiste en recordar literalmente partes del texto, sino que se trata de abstraer y parafrasear las ideas principales que recogen la información general del texto, tanto literal o textual como inferencial, generada a partir de los conocimientos de quien lea.

Núñez (2009) afirma que el procesamiento semántico lo construye la persona lectora, organizando los datos que el texto le aporta y el grado de comprensión dependerá de la fidelidad con que asimile los conceptos que el mensaje le transmite (competencia lingüística y conceptual), y la habilidad para organizar los conceptos evocados por la lectura en su mente, relacionándolos con sus experiencias previas. Esto le permitirá ampliar, completar, variar y demás, los conocimientos sobre el tema. El autor indica que, para lograrlo, quienes leen deben contar con las siguientes capacidades: diferenciar los grupos de letras dotadas de significado, saber prever lo que seguirá en la cadena escrita, reconocer el significado de los signos de puntuación y los espacios en blanco, captar globalmente el significado y la función de un enunciado, reconocer la aceptabilidad o inaceptabilidad semántica de una secuencia, identificar el significado de los elemento deíciticos, discriminar el significado de un 
lexema conocido con anterioridad y, finalmente, reconocer los lexemas cuyo significado se desconoce.

Los cuatro niveles de procesamiento del lenguaje que convergen en la comprensión lectora descritos anteriormente deberían estar contemplados en las pruebas de evaluación de la competencia lectora en los diferentes años escolares de primaria, para identificar necesidades educativas especiales en esas áreas. Sin embargo, en Costa Rica no existe un instrumento formal que tome en cuenta estos procesos lectores, razón por la cual surgió la idea de adaptar el Test de Lectura y Escritura en Español: LEE (Test LEE en adelante) para su posible uso en nuestro contexto escolar.

\section{Test de Lectura y Escritura en Español (LEE)}

EI Test LEE es un instrumento elaborado por un equipo de profesionales argentinos y españoles, dirigido por la Dra. Defior Citoler, es una batería compuesta por varias pruebas que permiten evaluar el rendimiento y las dificultades específicas de lectura y escritura. Ha sido elaborado atendiendo íntegramente a las particularidades lingüísticas del español castellano y el español americano, y consta de 7 pruebas: lectura de palabras; lectura de pseudopalabras; comprensión de palabras y frases; prosodia; comprensión de textos; escritura de palabras y escritura de pseudopalabras. También incluye 2 suplementarias: segmentación fonémica y lectura de letras. Cuenta con baremos que permiten determinar los logros medios esperables por año escolar (de $1^{\circ}$ a $4^{\circ}$ grado de la escuela primaria). En la última parte se brindan los criterios para la interpretación de las puntuaciones, ilustradas mediante la exposición de 3 casos, así como una guía para orientar a profesionales en las tareas de recuperación de las dificultades de lectura y escritura.

La validez convergente del Test LEE se obtuvo a partir de la correlación de esta batería con otras pruebas de probada validez que se utilizan para detectar alteraciones en el rendimiento de la lectura y la escritura, como son el ProLec (Cuetos, Rodríguez, Ruano, 1996) y el ProEsc (Cuetos, 2002). Para gran parte de las pruebas valoradas se encontraron correlaciones positivas y significativas entre el Test LEE y las otras medidas estudiadas.

Por otra parte, la validez discriminante se obtuvo a través de la comparación de las diferencias en el rendimiento de un grupo de estudiantes de población general y un grupo de estudiantes con diagnóstico de alteraciones en alguno de los proceso de lectoescritura. Tanto el rendimiento en las pruebas de lectura (de palabras y pseudopalabras) como en las 
de escritura (de palabras y pseudopalabras), así como la tarea de comprensión de textos discriminaron entre el estudiantado de ambas muestras.

En el mismo sentido, los estudios de confiabilidad del Test LEE arrojaron indicadores satisfactorios tanto acerca de la consistencia interna de los ítems que conforman todas las pruebas de la batería como de la confiabilidad test-pretest con un mes de intervalo entre las dos administraciones, y con la confiabilidad por división por mitades de las pruebas de lectura y escritura. En suma, las propiedades estudiadas del test indican que este instrumento constituye una herramienta adecuada para evaluar procesos vinculados a la lectoescritura en estudiantes de primero a cuarto grado de primaria.

Este instrumento fue utilizado en la tesis Eficacia de las Estrategias Pictofónicas en la enseñanza de la lectura inicial en Costa Rica: Un estudio Longitudinal, de la investigadora principal de este estudio, cuyo objetivo fue contrastar la efectividad en la enseñanza de la lectura de las Estrategias Pictofónicas, método que estimula un proceso dual de aprendizaje de la lectura mediante la asociación del grafema con el dibujo que lo representa (pictograma) y el sonido inicial de la palabra clave (fonema) en tres escuelas públicas (grupo experimental), frente al Método Ecléctico, aplicado en otras tres escuelas públicas (grupo cuasi control) del Núcleo Uno, Circuito 05 de la Región Educativa de Cartago, Costa Rica, entre los años 2011 y 2012.

Para demostrar las diferencias significativas en la competencia lectora entre el estudiantado instruido con uno y otro método, se realizó un estudio experimental de diseño pre-post con grupo cuasi control, aplicando subpruebas del Test LEE en febrero, junio y noviembre de 2010, cuyos resultados fueron a favor del método experimental (CarpioBrenes, 2011, Carpio-Brenes, 2014). En la última medición realizada en setiembre de 2011, se determinó que el desarrollo de la experticia lectora en estudiantes que fueron instruidos con las Estrategias Pictofónicas como el de mejor desempeño realizado en las ocho variables medidas (segmentación fonética, velocidad lectora, fluidez lectora, grupo consonántico -CCV-, descifrado, longitud polisílaba, calidad lectora y decodificación de palabras y pseudopalabras).

Con el rendimiento mostrado por el total de 333 estudiantes participantes en esa investigación y tras un análisis del contenido léxico del total de las subpruebas que comprende el Test LEE por parte de la investigadora principal, se pudo observar que el vocabulario empleado en ellas se ajusta a nuestro contexto escolar. Esta razón la motivó a 
proponer su adaptación para Costa Rica ante la ausencia de pruebas estandarizadas para la evaluación del desempeño lector en los diferentes años escolares.

\section{Metodología}

\subsection{Tipo de estudio}

Esta investigación se enmarca en un diseño instrumental con metodología cuantitativa, porque, de acuerdo con León y Montero (2003), pertenecen a esta categoría "todos los estudios encaminados al desarrollo de pruebas y aparatos, incluyendo tanto el diseño (o adaptación) como el estudio de las propiedades psicométricas de los mismos" (p. 799), y aquí lo que se pretende es validar el contenido léxico de una prueba extranjera para ajustarla a las particularidades lingüísticas del contexto educativo costarricense, mediante el análisis cuantitativo de los datos obtenidos por la triangulación de tres fuentes de información: estudiantes, docentes y expertos.

\subsection{Población y muestra}

Para alcanzar este propósito, se decidió utilizar como población meta para la prueba piloto a estudiantes de $1^{\circ}$ a $4^{\circ}$ de Educación General Básica del año 2013 del Circuito 09 de la Región Educativa de Cartago. Estos niveles escolares corresponden a los que evalúa el Test LEE.

Con respecto al cálculo y selección de la muestra de estudiantes, se utilizó como marco muestral la lista oficial de las escuelas públicas que administra el Circuito 09 de la Región Educativa de Cartago 2013, por escuela y nivel, facilitada en la oficina circuital. El cuadro se muestra a continuación. 
Cuadro 1.

Cantidad de estudiantes y grupos por escuela, según nivel Marco muestral

\begin{tabular}{|c|c|c|c|c|c|c|c|c|}
\hline \multirow{3}{*}{ Es cuela } & \multicolumn{8}{|c|}{ Nivel es colar } \\
\hline & \multicolumn{2}{|l|}{1} & \multicolumn{2}{|l|}{2} & \multicolumn{2}{|l|}{3} & \multicolumn{2}{|l|}{4} \\
\hline & es tudiantes & grupos & es tudi antes & grupos & estudiantes & grupos & es tudiantes & grupos \\
\hline Calle Mesén & 26 & 1 & 21 & 1 & 27 & 1 & 25 & 1 \\
\hline Villas de Ayarco & 87 & 3 & 73 & 3 & 71 & 3 & 109 & 4 \\
\hline San Francisco & 38 & 2 & 32 & 1 & 32 & 1 & 22 & 1 \\
\hline Calle Naranjo & 31 & 1 & 32 & 1 & 29 & 1 & 36 & 2 \\
\hline La Cima & 33 & 1 & 21 & 1 & 24 & 1 & 28 & 1 \\
\hline San Vicente & 59 & 3 & 58 & 3 & 75 & 3 & 77 & 3 \\
\hline Femando Terán & 132 & 5 & 113 & 6 & 114 & 5 & 133 & 6 \\
\hline Yerbabuena & 17 & 1 & 10 & 1 & 17 & 1 & 17 & 1 \\
\hline Moisés Coto & 70 & 3 & 73 & 3 & 67 & 2 & 70 & 3 \\
\hline Ricardo André & 44 & 2 & 40 & 2 & 54 & 2 & 44 & 2 \\
\hline Unidad Pedagógica & 67 & 3 & 60 & 3 & 89 & 3 & 95 & 4 \\
\hline M. Amelia Montealegre & 27 & 1 & 20 & 1 & 28 & 1 & 29 & 1 \\
\hline Carolina Bellelli & 72 & 2 & 54 & 2 & 55 & 2 & 71 & 2 \\
\hline Domingo Faustino & 17 & 1 & 20 & 1 & 25 & 1 & 21 & 1 \\
\hline El Carmen & 59 & 2 & 45 & 2 & 46 & 2 & 66 & 2 \\
\hline Santiago del Monte & 104 & 4 & 93 & 3 & 75 & 3 & 99 & 4 \\
\hline Central de Tres Ríos & 163 & 6 & 154 & 6 & 194 & 7 & 172 & 6 \\
\hline Calle Girales & 44 & 2 & 46 & 2 & 51 & 2 & 46 & 2 \\
\hline Quebrada del Fierro & 24 & 1 & 26 & 1 & 20 & 1 & 19 & 1 \\
\hline Total & 1114 & 44 & 991 & 43 & 1093 & 42 & 1179 & 47 \\
\hline
\end{tabular}

Fuente: Elaboración propia

La muestra se seleccionó estratificada por nivel (1ํa $4^{\circ}$ grado). Cada una de las muestras se obtuvo fijando un nivel de confianza del 95\% (precisión $Z_{\alpha / 2}$ ), un margen de error del $5 \%(\varepsilon)$. La fórmula utilizada para el cálculo muestral por estrato fue la siguiente:

$$
n_{0}=\frac{z_{\partial / 2}^{2} * p^{*}(1-p)}{\varepsilon^{2}}
$$

El tamaño de la muestra inicial se ajustó por finitud tomando en cuenta la matricula actual. La muestra aceptante quedó en 802 estudiantes, distribuidos por nivel, según se presenta en el Cuadro 2.

Cuadro 2.

Cantidad de estudiantes seleccionados por nivel

\begin{tabular}{|lccc|}
\multicolumn{1}{c}{ Nivel } & Total de estudiantes & Muestra & $\begin{array}{c}\text { Grupos } \\
\text { seleccionados }\end{array}$ \\
\hline Primer grado & 1114 & 201 & 8 \\
\hline Segundo grado & 991 & 197 & 10 \\
\hline Tercer grado & 1093 & 201 & 8 \\
\hline Cuarto grado & 1179 & 203 & 9 \\
\hline Total & $\mathbf{4 3 7 7}$ & $\mathbf{8 0 2}$ & $\mathbf{3 5}$ \\
\hline
\end{tabular}

Fuente: Elaboración propia 
De las 19 escuelas que presenta el Cuadro 1, se escogieron al azar 15 que, entre sus grupos, completaran la cantidad de la muestra aceptante. Quedaron fuera de la muestra las escuelas Ricardo André, María Amelia Montealegre, Domingo Faustino y El Carmen. De cada nivel escolar se escogieron entre 8 y 10 grupos completos mediante una selección aleatoria, resultando en algunas escuelas un solo grupo, y en aquellas que habían dos o más del mismo nivel, se escogió el grupo en el sitio y momento de la aplicación del instrumento.

Figura 1.

Condición de la muestra aceptante

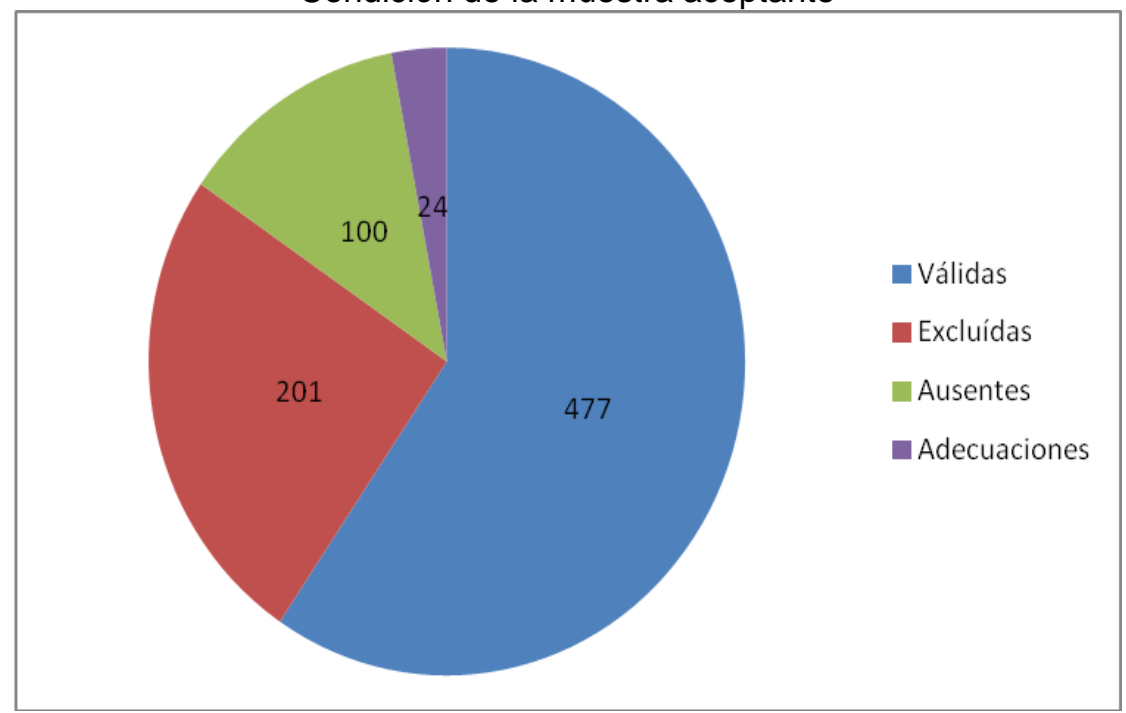

Fuente: Elaboración propia

Por una serie de imprevistos suscitados durante la aplicación de los textos en los meses de octubre y noviembre del 2013, que se muestran en la Figura 1, la muestra real para la validación del contenido de los textos de comprensión lectora quedó en 477 estudiantes, porque 201 estudiantes de primer grado fueron excluidos del estudio por no haber alcanzado un nivel de decodificación lectora aceptable; 100 estudiantes estaban ausentes el día que se presentaron los textos; y 24 estudiantes con adecuación curricular significativa no sabían leer.

\subsection{Instrumentos}

Como se mencionó antes, el Test LEE fue diseñado por un grupo de expertos de Argentina y España. Está organizado en dos grandes componentes de la lectura: el reconocimiento de palabras y la comprensión lectora, subdivididos en las siguientes subpruebas: 
1. Lectura de palabras

2. Lectura de pseudopalabras

3. Comprensión de palabras y frases

4. Prosodia

5. Comprensión de textos

6. Escritura de palabras

7. Escritura de pseudopalabras

La lectura y escritura de palabras y la lectura y escritura de pseudopalabras (pruebas 1, 2, 6 y 7 respectivamente) evalúan en el estudiantado el nivel de procesamiento léxico, que implica la habilidad de estas personas lectoras de reconocer las palabras utilizando dos vías para su acceso: la vía léxica (directa o visual), que implica un conocimiento inmediato de palabras conocidas, es decir, que ya hayan sido procesadas anteriormente y que están guardadas en el léxico mental; y la vía fonológica (indirecta o subléxica) que es el mecanismo que convierte las palabras desconocidas o las pseudopalabras en sonidos, y las secuencias de sonidos en palabras, mediante la aplicación de las reglas de correspondencia grafema-fonema (Coltheart, 2007)

Las pruebas 3, 4 y 5, correspondientes a la valoración de la comprensión de palabras y frases, de la prosodia y de la comprensión de textos. Evalúan los procesamientos sintácticos y semánticos desarrollados en el estudiantado, es decir, la capacidad que tienen de identificar las estructuras gramaticales y extraer el mensaje de la frase, oración o texto que leen.

En este artículo se reporta la validez de contenido léxico de los seis textos que se proponen para la evaluación de la comprensión lectora (prueba 5). Estos se dividen por niveles de complejidad en tres textos para primero y segundo grado, y tres para tercero y cuarto grado. La prueba está dividida en tres subpruebas, la primera corresponde a seis preguntas de tipo literal e inferencial para cada uno de los seis textos; la segunda es la identificación del título de cada texto de entre 4 opciones, y la tercera se refiere al reconocimiento de la idea principal en cada texto de entre 4 enunciados.

\subsection{Procedimiento}

La validación del contenido de los textos para la comprensión lectora del Test LEE se realizó en tres vías. La primera consistió en la señalización, por parte del alumnado de la 
muestra, de aquellas palabras que le resultaban desconocidas en los textos; la segunda fue el reconocimiento del vocabulario difícil en esos textos por parte de docentes del grupo muestral; y el tercero implicó la identificación de las palabras descontextualizadas en los textos por parte de cinco personas expertas en temas relacionados con la enseñanza y evaluación de la lengua.

Durante el 2013 se ejecutaron las actividades orientadas a la identificación de las dificultades léxicas de los textos de comprensión del Test LEE por parte del estudiantado de la muestra seleccionada y sus docentes. El trabajo de campo que se realizó en las escuelas fue coordinado por las investigadoras con el Supervisor de Centros Educativos del Circuito 09 y la dirección de cada una de las diferentes instituciones educativas. Estas dos instancias dieron su aprobación para presentar los textos como una actividad regular y académica a todo el grupo dentro de la clase, por esa razón no se entregó carta de consentimiento informado a los padres y las madres de familia del estudiantado.

Los textos fueron entregados a cada estudiante y a su docente por las investigadoras, quienes tardaron aproximadamente 50 minutos en cada grupo para indicarle a las personas participantes que leyeran cada uno de los tres textos que conforman las pruebas y marcaran con lápiz las palabras desconocidas para ellas. Los textos de cada estudiante fueron organizados en carpetas, por institución y sección. Los textos de docentes se reunieron en dos apartados, los correspondientes a los niveles de primero y segundo grado, y los de tercero y cuarto grado. La mayoría de los datos del estudiantado fue digitados en el programa estadístico CSPro.

En el 2014 se obtuvo el criterio de las personas expertas en relación con el vocabulario que consideraban difícil de comprender en los textos, por su poco uso en el contexto costarricense. Se escogieron cinco profesionales relacionados con la enseñanza y evaluación de la lengua:

- Especialista en enseñanza de la lengua, asesora regional de Español de la Región Educativa de Cartago.

- Especialista en problemas de aprendizaje de la lectura y la escritura, docente e investigadora de la Universidad Estatal a Distancia.

- Especialista en filología, docente e investigadora de la Universidad de Costa Rica.

- Especialista en evaluación, docente e investigadora de la Universidad de Costa Rica.

- Especialista en didáctica de la lectoescritura, docente e investigadora de la Universidad de Costa Rica. 
A cada una de estas personas se le entregó un folder que incluía una carta explicando el propósito del estudio y dando la consigna de qué hacer con los textos, una hoja con unos recuadros para incluir las palabras que seleccionaran como difíciles y recomendaran sinónimos y una constancia de que los textos los consideraban pertinentes para el contexto educativo nacional. Los datos se registraron en Excel para su posterior análisis.

\subsection{Análisis de los datos}

Para el análisis de los datos se decidió utilizar la triangulación, porque se obtuvo información de tres fuentes diferentes: estudiantes, docentes y expertos. En el programa estadístico CSPro, se incorporaron todas las palabras que fueron marcadas por estos tres grupos; luego, se realizó el análisis de frecuencia para identificar cuáles de esas palabras habían sido escogidas por la mayor cantidad de personas participantes de cada grupo.

Después, se compararon las frecuencias de las palabras escogidas por el grupo de estudiantes, el grupo docente y el grupo de expertos en los distintos niveles escolares según los textos que les correspondieron. Esto se debe a que para los niveles de primero y segundo, el Test LEE propone 3 textos que son diferentes a los que se plantean para los niveles de tercero y cuarto grado.

Para el análisis de los datos se estableció, como criterio de selección de las palabras que se considerarían de dificultad léxica, aquellas que habían alcanzado las frecuencias más altas en cada uno de los tres grupos participantes. Finalmente, se analizaron cuáles de esas palabras que obtuvieron mayor frecuencia de selección por nivel escolar, coincidían entre los tres grupos, y estas fueron las que se consideraron como las palabras de dificultad léxica, a las que se les debe proponer un sinónimo en el instructivo que se pretende incorporar en el Test LEE para su aplicación en Costa Rica.

\section{Análisis de los datos y resultados}

Como los datos para la validación del contenido de los textos para la comprensión lectora del Test LEE se obtuvieron de tres fuentes, los resultados se exponen en tres apartados: estudiantes, docentes y expertos.

\subsection{Estudiantes}

A pesar de que el trabajo de campo con los grupos de primer grado se realizó en la última semana de noviembre asumiendo que ya sabrían leer, al presentarles los textos a las 
ocho docentes de este nivel todas manifestaron que sus estudiantes aún no habían adquirido la decodificación y fluidez lectora suficiente como para enfrentarse a los tres textos que propone el Test LEE. Por ello se decidió excluir este nivel del estudio, pero sí dejar a sus docentes en la validación del contenido de los textos del nivel primero y segundo grado.

De acuerdo con el cálculo de la muestra aceptante, se esperaba obtener los datos de 197 estudiantes de segundo grado, 201 de tercer grado y 203 de cuarto grado; no obstante, el día que se recogió la información varios estudiantes se ausentaron. Los motivos expuestos por sus docentes fueron: deserción, traslado, ausencia injustificada, ausencia justificada y en ciertas escuelas estaban en período de exámenes finales, por lo que algunos estudiantes no asistieron a lecciones porque estaban eximidos.

Figura 2.

Cantidad de niños y niñas participantes, según nivel escolar

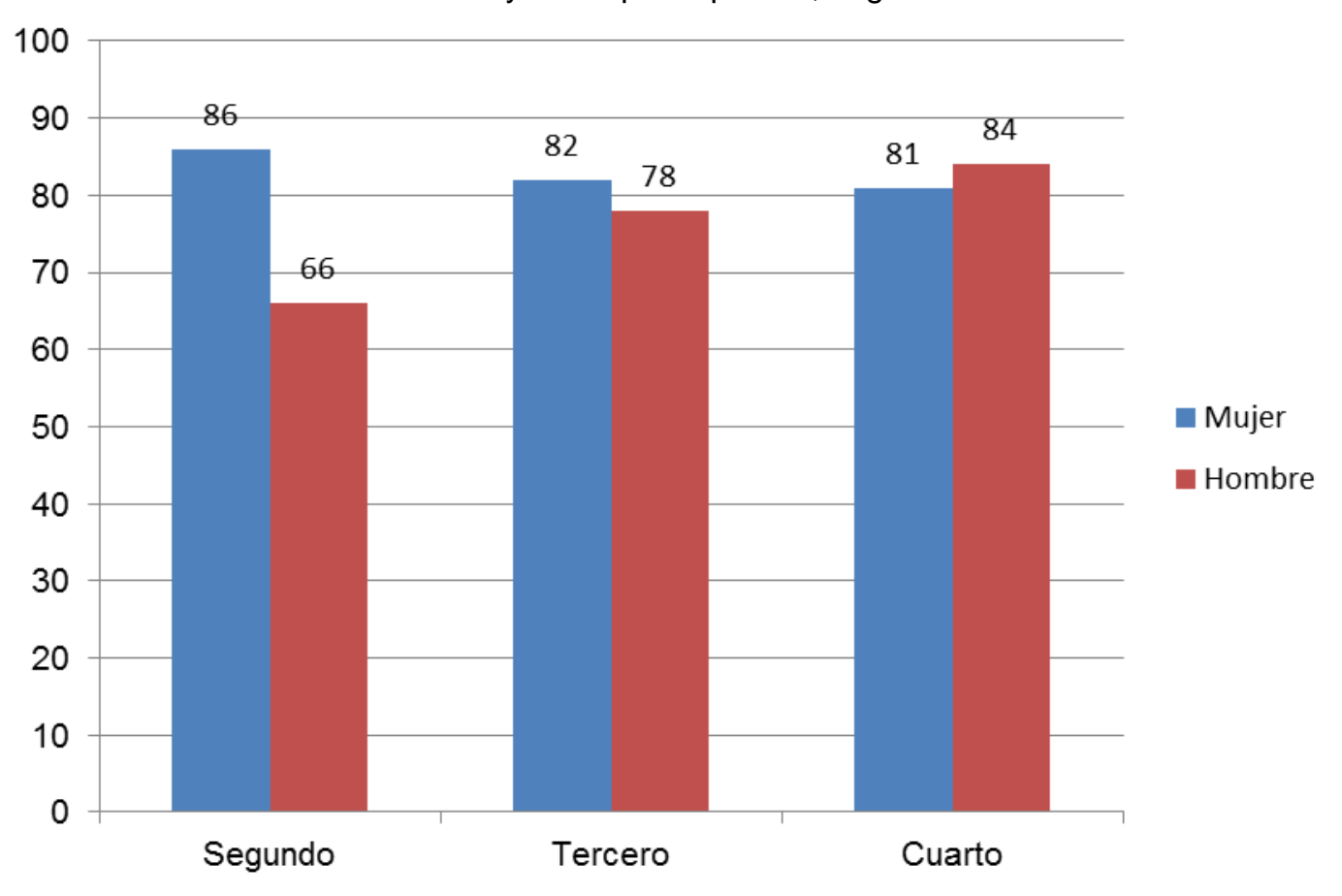

Fuente: Elaboración propia

De acuerdo con la Figura 2, la muestra real de 477 estudiantes quedó distribuida, por nivel y por sexo, de la siguiente manera: 152 en segundo grado, con 86 mujeres y 66 hombres; 160 estudiantes en tercer grado, con 82 mujeres y 78 hombres; y 165 estudiantes en cuarto grado, con 81 mujeres y 84 hombres. 
Figura 3.

Palabras consideradas desconocidas por estudiantes de segundo grado

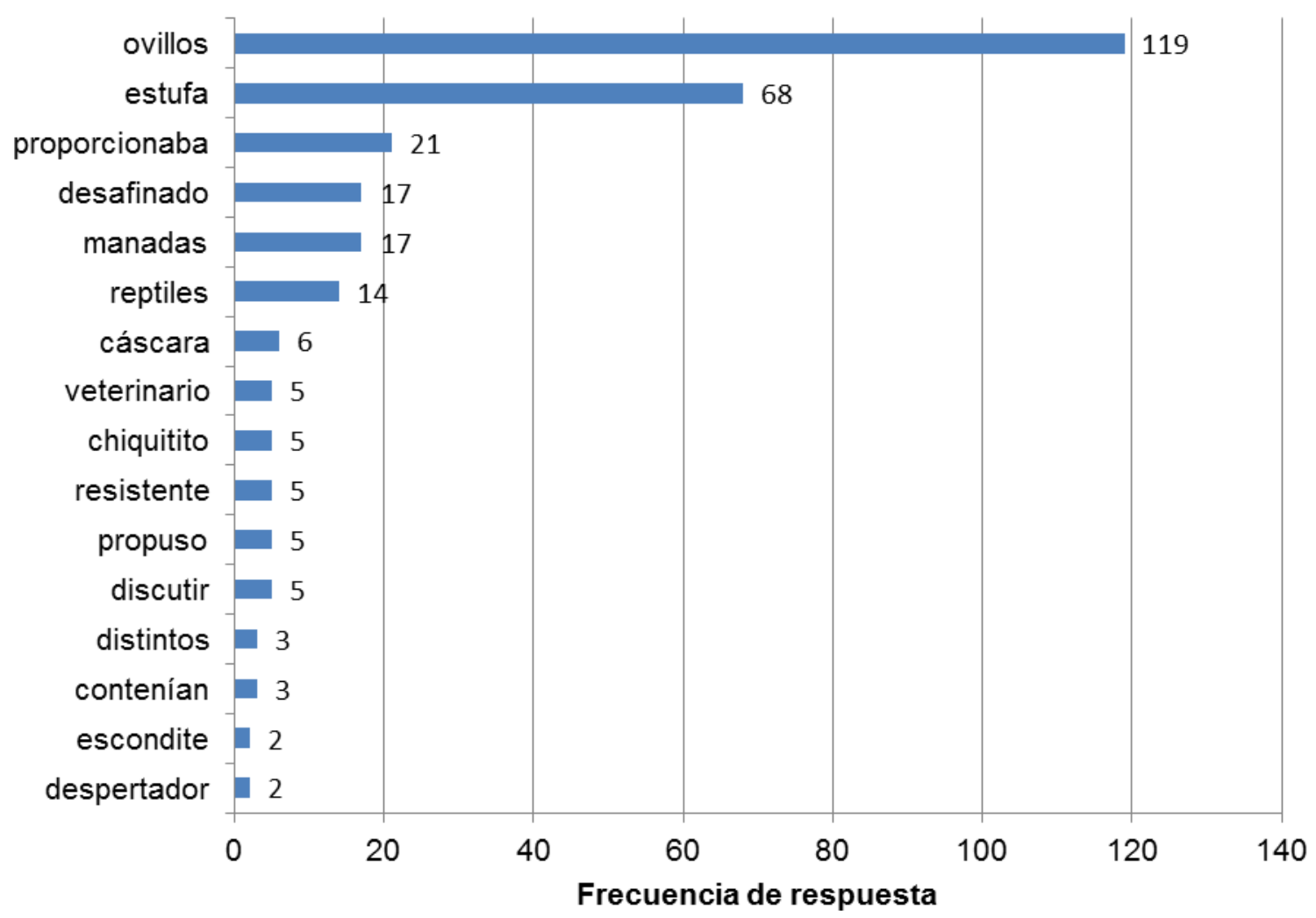

Fuente: Elaboración propia

Como se observa en la Figura 3, 16 palabras de los tres textos de comprensión lectora fueron escogidas como desconocidas por estudiantes de segundo grado. De ellas, se destacan las dos que tienen mayor frecuencia de selección por parte del estudiantado, que son: la palabra ovillos con 119 estudiantes de la muestra que la subrayaron; y la palabra estufa, con 68 estudiantes que la escogieron. Ambas palabras pertenecen al primer texto de los niveles de primero y segundo grado. 
Figura 4.

Palabras consideradas desconocidas por estudiantes de tercer grado

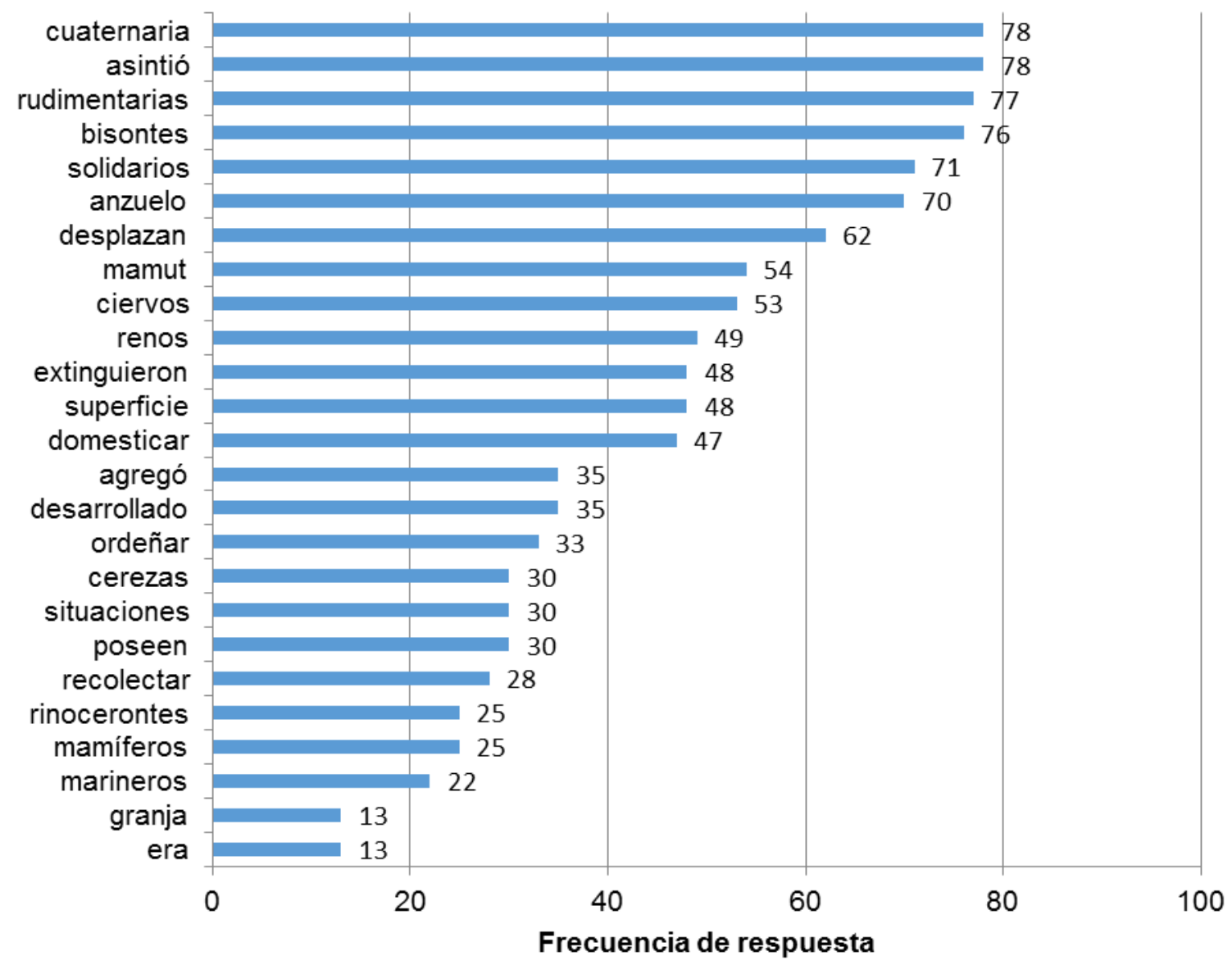

Fuente: Elaboración propia

La Figura 4 muestra las 25 palabras que estudiantes de tercer grado consideraron desconocidas en los textos que corresponden a los niveles de tercero y cuarto grado. Resaltan en el gráfico las seis palabras que más de 70 estudiantes de los 160 de este nivel subrayaron en los textos como desconocidas: cuaternaria (78), asintió (78), rudimentarias (77), bisontes (76), solidarios (71) y anzuelo (70). 
Figura 5.

Palabras consideradas desconocidas por estudiantes de cuarto grado

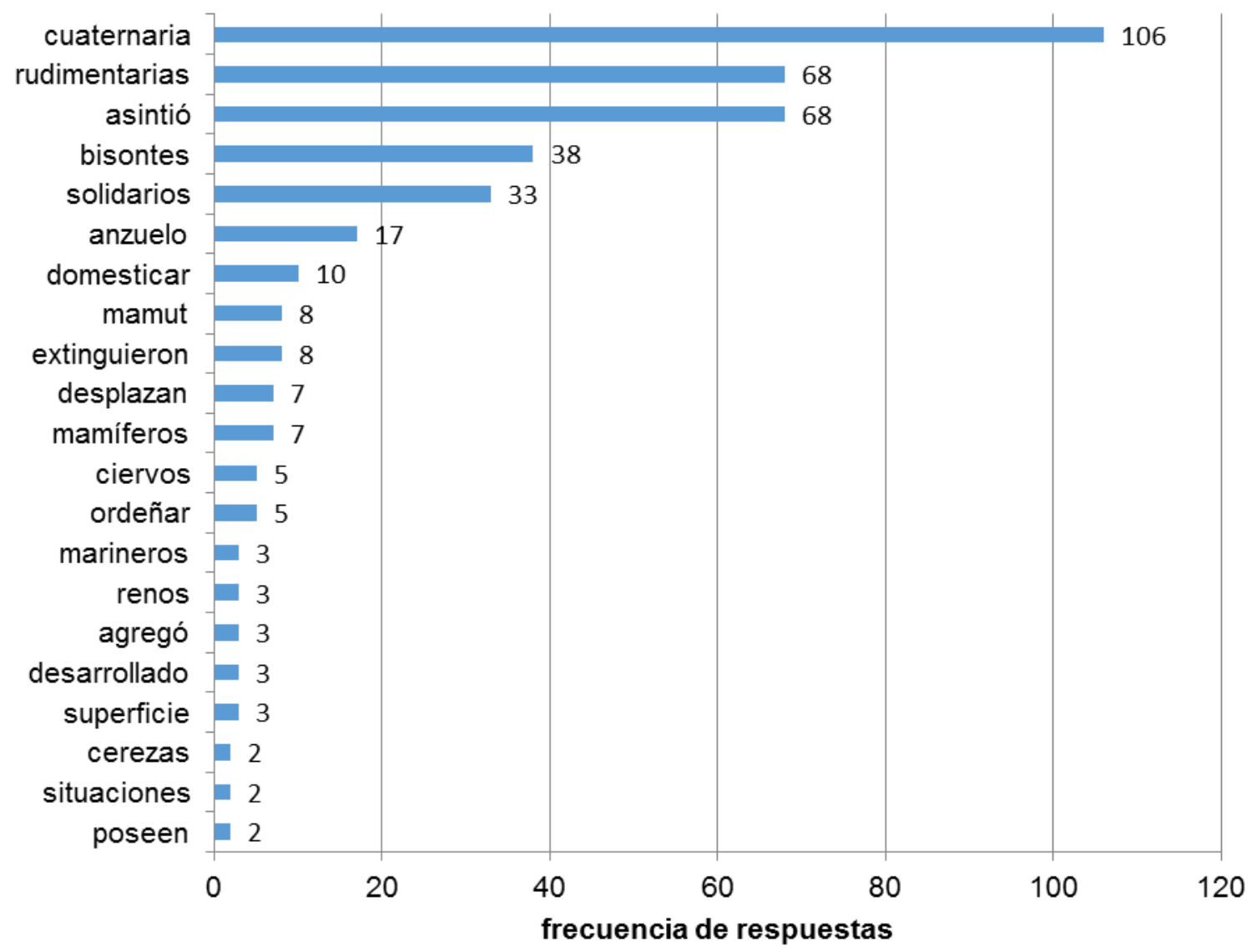

Fuente: Elaboración propia

Con respecto a las palabras subrayadas por estudiantes de cuarto grado, la Figura 5 muestra cómo las palabras cuaternaria (106), rudimentarias (68) y asintió (66) fueron escogidas por más de 65 estudiantes como desconocidas para este nivel conformado por 165 estudiantes.

Al analizar los datos suministrados por estudiantes de los tres grados escolares, se puede decir que de los textos del nivel de primero y segundo grado, las palabras que la mayoría de estudiantes de segundo grado considera desconocidas son ovillo y estufa, ambas correspondientes a la primera lectura de las tres propuestas.

De los textos del nivel correspondiente a tercero y cuarto grado, el estudiantado coincidió en las tres palabras desconocidas con mayor frecuencia de selección: cuaternaria, rudimentaria y asintió. Las dos primeras palabras se encuentran en el segundo texto y la palabra cuaternaria en el tercer texto. 


\subsection{Docentes}

La muestra real de 477 estudiantes quedó distribuida en 35 grupos, 8 de primer grado, 10 de segundo grado, 8 de tercer grado y 9 de cuarto grado, cada grupo a cargo de docentes diferentes.

Se le entregó a cada persona docente los tres textos de acuerdo al grado que cursaban sus estudiantes, y se les pidió que subrayaran las palabras que les podían resultar de difícil comprensión por ser un término poco frecuente en nuestro contexto escolar. Los resultados se muestran en las siguientes figuras.

Figura 6.

Palabras consideradas desconocidas por docentes de primer grado

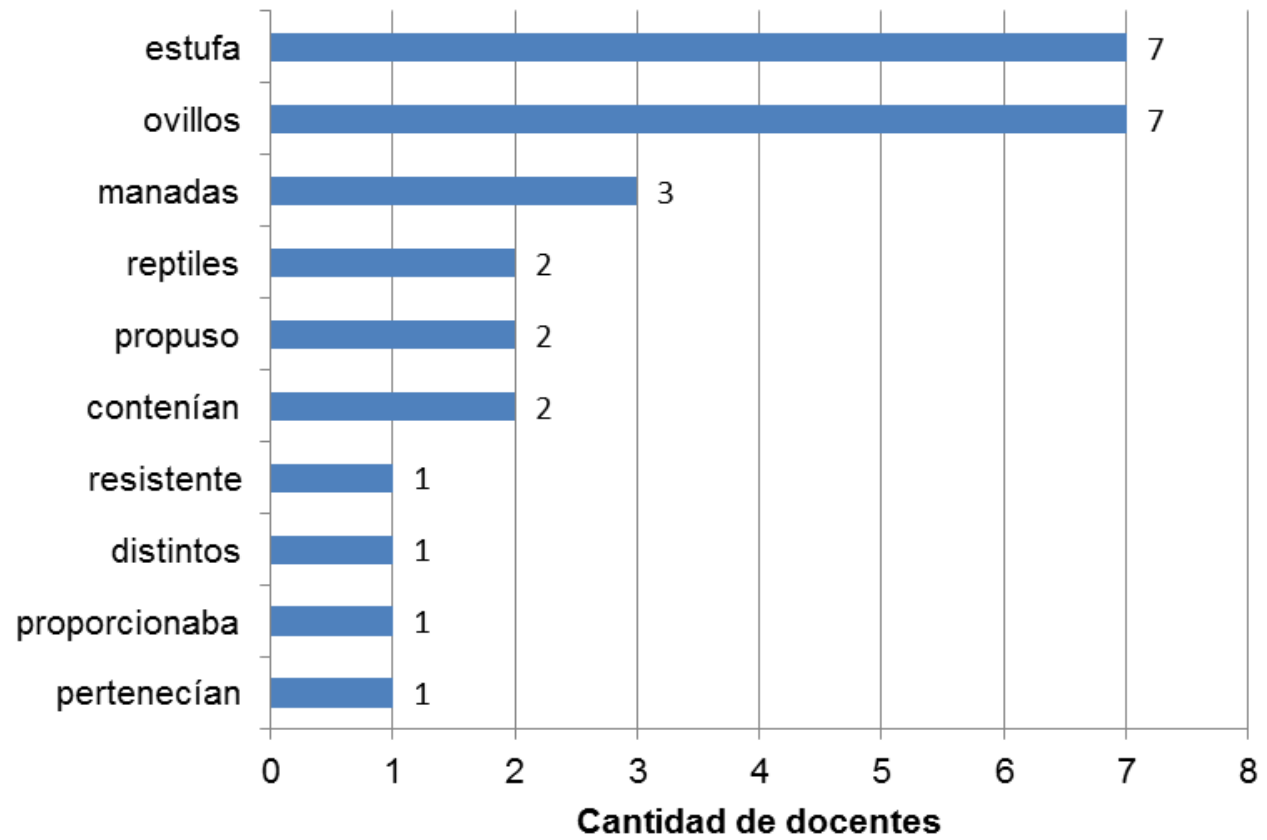

Fuente: Elaboración propia. (2016)

Como se mencionó antes, a pesar de que se excluyó a los grupos de estudiantes de primer grado, se consideró el criterio de sus docentes para la validez de contenido léxico de los textos de los niveles de primero y segundo grado. La Figura 6 muestra cómo de los ocho docentes que imparten primero, siete de ellos y ellas escogieron las palabras estufa y ovillos como de uso poco frecuente en nuestro contexto. Menos de la mitad de docentes indicaron 
otras palabras como manadas (3), reptiles (2), propuso (2), contenían (2), y una sola persona docente agregó resistente, distintos, proporcionaba y pertenecían.

Figura 7.

Palabras consideradas desconocidas por docentes de segundo grado

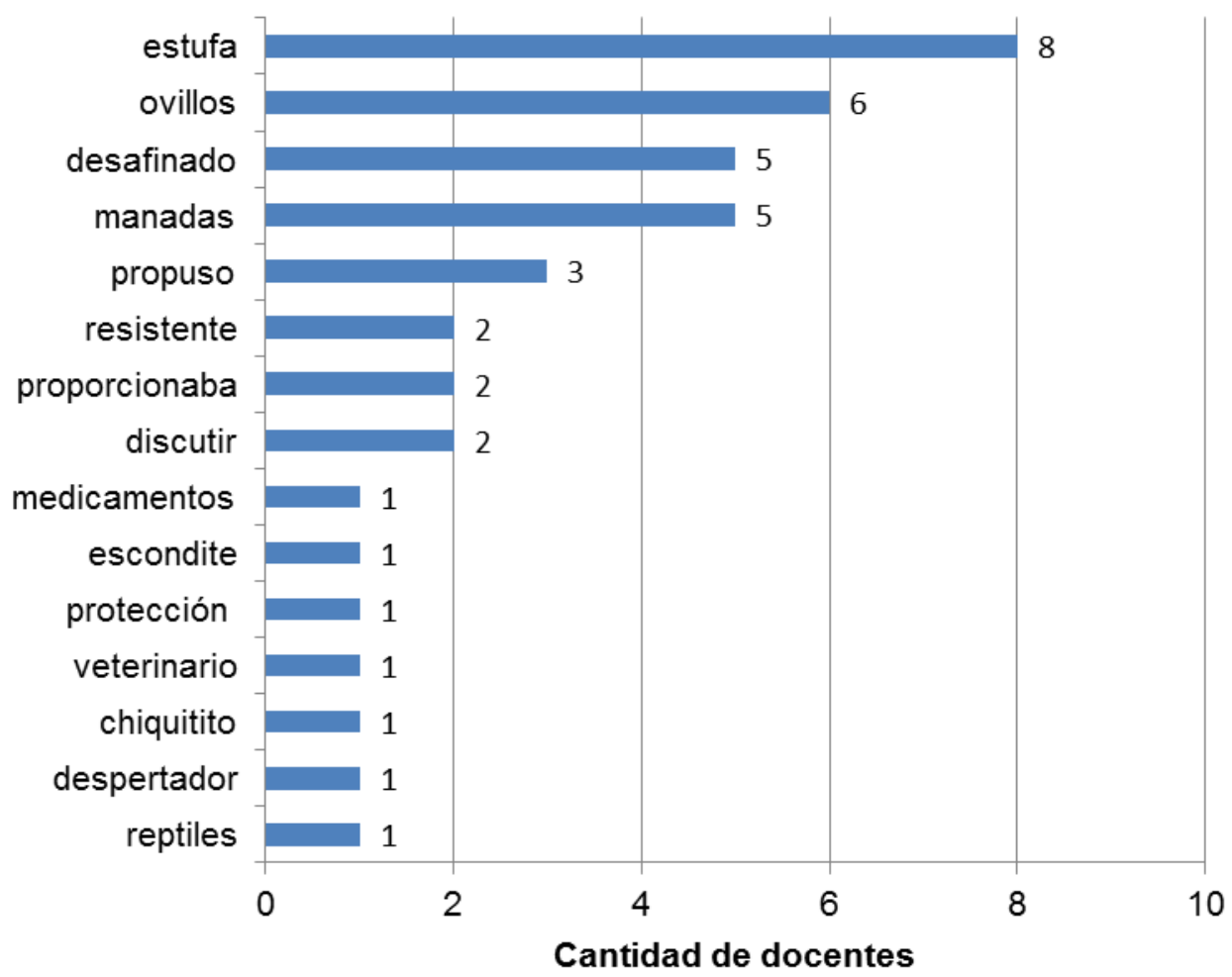

Fuente: Elaboración propia. (2016)

Como se muestra en la Figura 7, de 10 docentes que imparten segundo grado, la mayoría consideró las palabras estufa (8) y ovillos (6) como palabras que podrían ser difíciles para sus estudiantes. La mitad incluyó las palabras desafinado y manadas, y pocos de ellos y ellas incluyeron otras palabras, como tres docentes que subrayaron la palabra propuso; dos docentes indicaron los vocablos resistente, proporcionaba y discutir; una persona indicó los términos medicamentos, escondite, protección, veterinario, chiquitito, despertador y reptiles. 
Figura 8.

Palabras consideradas desconocidas por docentes de tercer grado

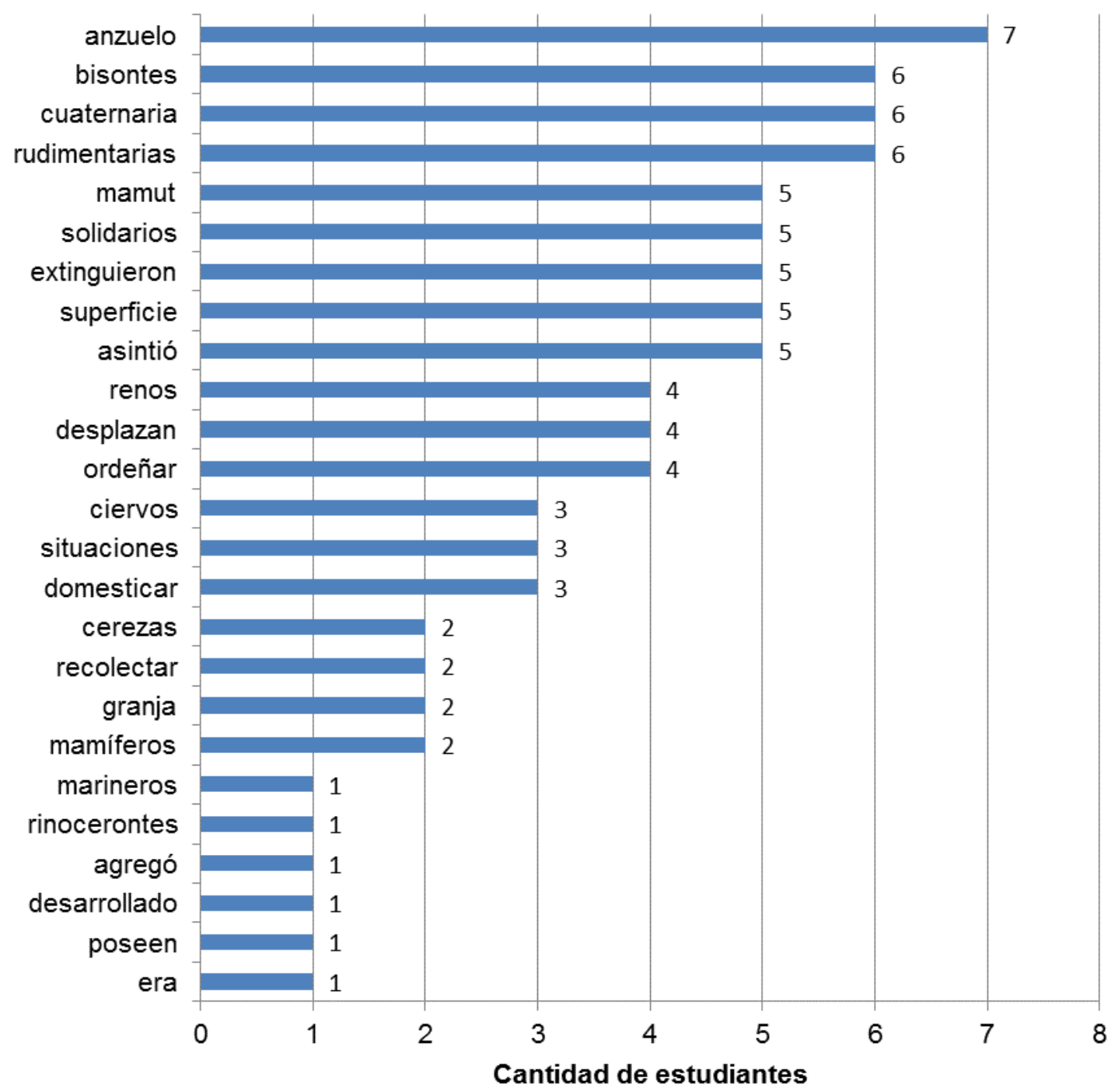

Fuente: Elaboración propia

En la Figura 8 se observa cómo el grupo de docentes de tercer grado escogió 25 palabras como desconocidas para sus estudiantes. La mayoría subrayó las palabras anzuelo (7), bisontes (6), cuaternaria (6) y rudimentarias (6) como las más difíciles. Cinco docentes incluyeron las palabras mamut, solidarios, extinguieron, superficie y asintió; cuatro subrayaron renos, desplazan y ciervos; otros tres docentes indicaron ciervos, situaciones, domesticar; dos anotaron cerezas, recolectar, granja, mamíferos; y un docente agregó otras palabras como marineros, rinocerontes, agregó, desarrollado, poseen y era. 
Figura 9.

Palabras consideradas desconocidas por docentes de cuarto grado

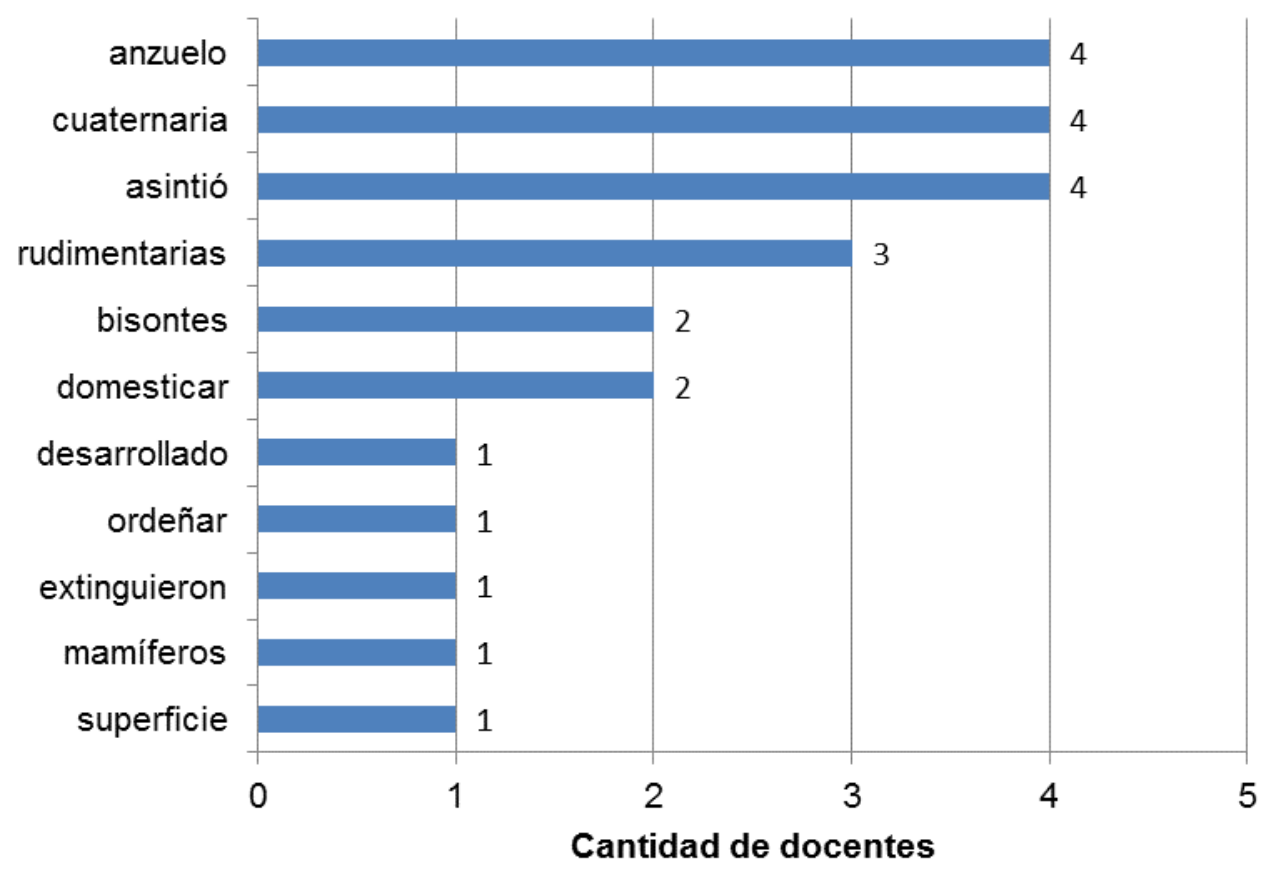

Fuente: Elaboración propia

El grupo docente de cuarto grado escogió 11 palabras como de difícil comprensión para sus estudiantes. La Figura 9 destaca que de nueve docentes de este nivel, cuatro subrayaron las palabras anzuelo, cuaternaria y asintió como las más difíciles; tres indicaron la palabra rudimentarias; dos las palabras bisontes y domesticar; y una persona incluyó los vocablos desarrollado, ordeñar, extinguieron, mamíferos y superficie.

Al analizar los datos suministrados por el grupo de 35 docentes, se puede decir que quienes imparten primero y segundo grado coinciden en que las dos palabras que pueden ser de difícil comprensión para sus estudiantes por el poco uso en el contexto costarricense son ovillos y estufa. Por su parte, el grupo de docentes de tercero y cuarto grado coincidió en tres palabras con mayor frecuencia: anzuelo, cuaternaria y rudimentarias.

\subsection{Expertos}

Al grupo de expertos, conformado por cinco especialistas en el campo de la enseñanza o evaluación de la lengua, se les entregó los seis textos que evalúan la comprensión lectora del Test LEE, y se les pidió que subrayaran las palabras que les podían resultar de difícil 
comprensión a los y las estudiantes costarricenses, por ser vocabulario poco frecuente en nuestro contexto escolar. Los resultados se muestran a continuación.

Figura 10.

Palabras consideradas desconocidas por el grupo de expertos

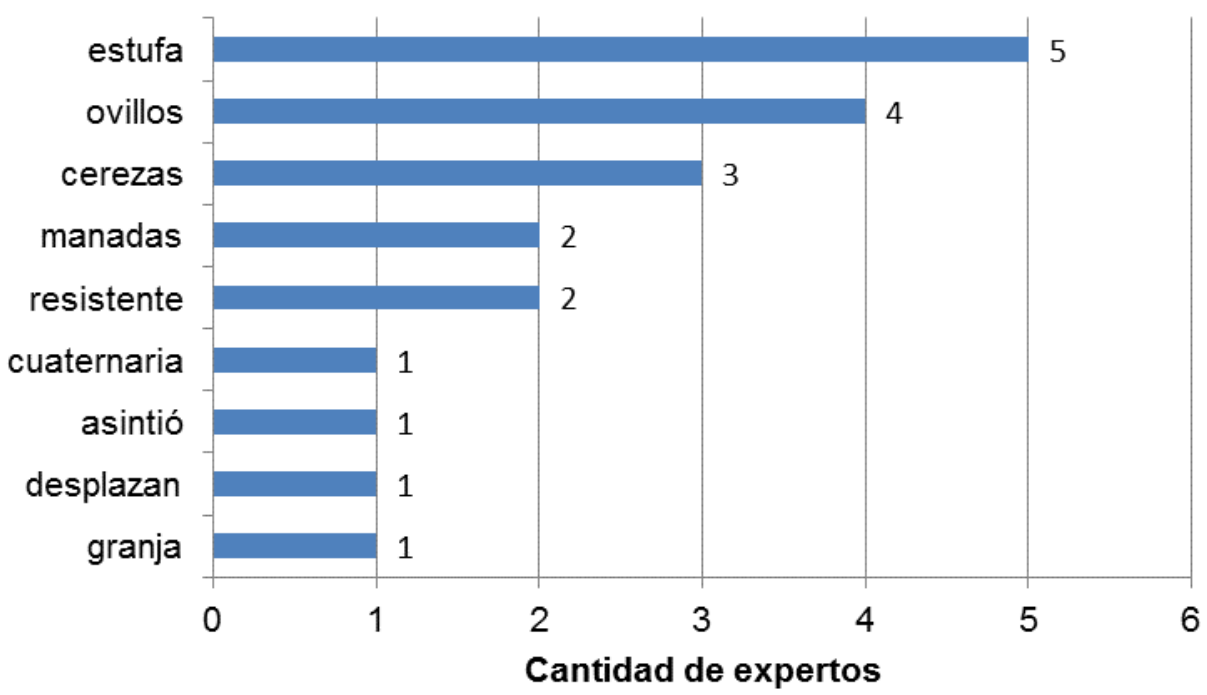

Fuente: Elaboración propia

La Figura 10 muestra que cinco de las expertas consideraron estufa y cuatro de ellas escogieron ovillos como las palabras más descontextualizadas, ambas son del texto 1 de los niveles de primero y segundo grado. Cuatro personas señalaron el vocablo cerezas que es del texto 2 de los niveles de tercero y cuarto grado. Dos indicaron manadas y resistente que están en el texto 2 de los niveles primero y segundo grado; y una persona marcó las palabras cuaternaria (texto 3 del nivel de primero y segundo grado tercero y cuarto grado), asintió (texto 1 del nivel de tercero y cuarto grado) desplazan (texto 1 del nivel de tercero y cuarto grado) y granja (texto 3 del nivel de primero y segundo grado) como difíciles.

Al analizar el criterio de la mayoría de personas expertas, se puede decir que las palabras consideradas de difícil comprensión para el estudiantado, por su poco uso en nuestro contexto costarricense, son estufa, ovillos y cerezas.

\section{Discusión y conclusiones}

Tres fueron los objetivos específicos que guiaron este estudio: la identificación de las diferencias léxicas de los textos, la adaptación de estas al vocabulario costarricense, y el diseño del instructivo que se utilizará cuando se apliquen las pruebas de comprensión lectora 
en la segunda parte de la investigación. Con base en los datos suministrados por las tres fuentes de esta investigación, para el cumplimiento del primer objetivo específico se obtuvo que:

- Para el estudiantado de segundo grado, las palabras difíciles fueron ovillo y estufa, ambas correspondientes a la primera lectura del nivel de primero y segundo grado. El grupo de tercero y cuarto grado consideró las palabras cuaternaria, rudimentaria $y$ asintió como complicadas, siendo las dos últimas del segundo texto y la palabra cuaternaria del tercer texto del nivel de tercero y cuarto grado.

- El grupo docente de primero y segundo grado consideró que las palabras que pueden ser de difícil comprensión para sus estudiantes por el poco uso en el contexto costarricense son ovillos y estufa. Quienes son docentes de tercero y cuarto grado coincidieron en tres palabras con mayor frecuencia: anzuelo, cuaternaria y rudimentarias.

- El grupo de expertos concordaron en tres palabras como descontextualizadas en los textos: estufa, ovillos y cerezas.

- Al relacionar la información, se encuentra coincidencias en la opinión del grupo de estudiantes de segundo grado, el grupo docente de primero y segundo grado y el grupo de personas expertas, pues la mayoría en los tres grupos consideran que las palabras de difícil comprensión en los textos de los niveles de primero y segundo grado son ovillo y estufa.

- Entre estudiantes de tercero y cuarto grado, sus docentes de estos niveles, y el grupo de personas expertas, coincide solo la palabra cuaternaria como difícil en los textos de los niveles de tercero y cuarto grado. La palabra rudimentarias fue señalada por estudiantes y docentes, pero no por las personas expertas. Quienes son docentes de estos niveles incluyen la palabra anzuelo como otro término difícil y el alumnado de ambos niveles indica que asintió es una palabra desconocida.

- La tercera palabra con mayor frecuencia de selección por parte del grupo de personas expertas fue cereza, de los textos del nivel de tercero y cuarto grado; sin embargo, de 325 estudiantes de tercero y cuarto grado, sólo 32 indicaron esta palabra como desconocida. 
De esta forma, se podría decir que para que los textos de comprensión lectora del Test LEE sean utilizados en Costa Rica, hay que hacer referencia a un sinónimo ante las siguientes palabras: ovillos, estufa, cuaternaria, rudimentarias, anzuelo y asintió.

El resto de palabras que fueron subrayadas por el grupo de estudiantes, docentes y expertos no se consideraron relevantes para la validez de contenido léxico, porque la intensión del estudio era identificar las palabras que podían presentar dificultad en su comprensión por ser de poco uso en nuestro contexto costarricense, es decir, complejidad léxica, que se refiere al conjunto de palabras de un idioma o una región, y no semántica, que implica el significado de las palabras, como sucede con el vocabulario del tercer texto del nivel de tercero y cuarto grado, cuyo tema se relaciona con las características de la era cuaternaria.

Se considera que, de las seis palabras que resultaron con mayor frecuencia de selección como palabra desconocida, cumplen el criterio de complejidad léxica los términos ovillos, estufa y asintió, porque éstos no son parte de nuestro lenguaje cotidiano, sino que en su lugar, en Costa Rica se dice lana, chimenea y aceptó. Las otras tres palabras resultan complejas por su significado, que pueden acceder a él si hay una adecuada comprensión del texto, pero se incluyen por la cantidad de estudiantes que las escogieron.

Para cumplir con el segundo objetivo de este estudio, que era adaptar este léxico al contexto costarricense, al grupo de expertos se le pidió sugerir un sinónimo a las palabras que consideraron difíciles en los seis textos, de tal manera que se utilizarían como parte de la adaptación de la prueba a nuestro contexto costarricense. El Cuadro 3 muestra el resultado de esta solicitud.

Cuadro 3.

Sinónimos sugeridos por personas expertas

\begin{tabular}{|cc|}
\hline Palabra del texto & $\begin{array}{c}\text { Sinónimo sugerido } \\
\text { bollas }- \text { rollos }\end{array}$ \\
\hline estufa & cocina - chimenea \\
\hline asintió & aceptó \\
\hline era cuaternaria & era de hielo \\
\hline rudimentarias & simples \\
\hline anzuelo & trampa \\
\hline
\end{tabular}

Fuente: Elaboración propia

El diseño del instructivo que se utilizará cuando se apliquen las pruebas de comprensión lectora en la segunda parte de la investigación, que es el tercer objetivo específico de la investigación, fue adjuntado en el informe general de este estudio. 
La limitación que se presentó durante la realización de esta investigación fue que el estudiantado de primer grado no supiera leer en el mes de noviembre. Esto afectó la cantidad de la muestra seleccionada. Tal hecho tendrá que analizarse profundamente para la realización de la segunda parte de este trabajo, donde se establecerá la confiabilidad de la prueba.

\section{Referencias}

Carpio-Brenes, María. (2011). Estrategias Pictofónicas. Un estudio comparativo de eficacia en la enseñanza de la lectura inicial para el primer grado de Educación general Básica en seis escuelas públicas en Cartago. Actualidades Investigativas en Educación, 11(2), 1-33. DOI: http://dx.doi.org/10.15517/aie.v11i2.10186

Carpio-Brenes, María. (2014). Efectividad de las estrategias pictofónicas en la adquisición de la decodificación lectora en estudiantes costarricenses. Actualidades Investigativas en Educación, 14(1), 1-34. DOI: http://dx.doi.org/10.15517/aie.v14i1.13215

Coltheart, Max. (2007). Modeling Reading: The Dual-Route Approach. En Margaret Snowling y Charles Hulme (Eds.), The Science of reading a Handbook (pp. 6-23). Estados Unidos: Blackwell Publishing.

Costa Rica, Ministerio de Educación Pública. (2012). Programme for International Student Assessment 2012. Recuperado de http://www.dgec.mep.go.cr/deac/pisa/pisainformacion

Costa Rica, Ministerio de Educación Pública. (2014). Programa de Estudios de Español. I Ciclo Educación General Básica. Recuperado de http://www.mep.go.cr/programaestudio?term node tid depth=3224

Cuetos, Fernando, Rodríguez, Blanca y Ruano, Elvira. (1996). Evaluación de los procesos lectores. ProLec. España: T.E.A. Ediciones S.A.

Cuetos, Fernando. (2002). ProEsc. Evaluación de los procesos de escritura. España: T.E.A. Ediciones S.A.

Cuetos, Fernando. (2008). Psicología de la lectura. España: Wolters Kluwer.

De Klerk, Louis y Simons, Peter. (1989). Estudio de los procesos metacognitivos de la comprensión de lectura. España: Fundación Germán Sánchez.

Defior, Sylvia y Serrano, Francisca. (2011). Procesos Fonológicos Explícitos e Implícitos, Lectura y Dislexia. Revista Neuropsicología, Neuropsiquiatría y Neurociencias, 11(1), 79-94.

Defior, Sylvia, Fonseca, Liliana, Gottheil, Bárbara, Aldrey, Adriana, Jiménez, Gracia, Pujals, María, Rosa, Graciela y Dolores, Francisca. (2006). LEE Test de lectura y escritura en español. Buenos Aires: Paidós. 
García, Emilio. (1993). La comprensión de textos. Modelo de procesamiento y estrategias de memoria. Revista Didáctica, 87-113. http://revistas.ucm.es/index.php/DIDA/article/viewFile/DIDA9393110087A/20216

Herrera, Lucía y Defior, Sylvia. (2005). Una Aproximación al Procesamiento Fonológico de los Niños Prelectores: Conciencia Fonológica, Memoria Verbal a Corto Plazo y Denominación. Psykhe, 14(2), 81-95

Huerta, Elena, y Matamala, Antonio. (1995). Tratamiento y prevención de las dificultades lectoras. Madrid: Visor Distribuciones S.A.

León, Ignacio y Montero, Orfelio. (2003). Métodos de Investigación en Psicología y Educación. Madrid: McGrawHill.

Lozano, Luis y Lozano, Manuel. (1999). Evaluación y tratamiento de la dislexia fonológica. Revista Aula Abierta, (74), 131-150.

McGuinness, Diane. (1999). A Scientific Revolution in Reading. Why Our Children Can't Read and What We Can Do About It. New York: Simon \& Schuster, Inc.

Núñez, Pilar. (2009). Los procesos de la comprensión lectora. Recuperado de https://es.scribd.com/doc/19345363/Teoria-Comprension-Lectora-Pilar-Nunez-Delgado

Organización de las Naciones Unidas para la educación, la Ciencia y la Cultura. (2008). SERCE: Segundo Estudio Regional Comparativo y Explicativo. Recuperado de http://unesdoc.unesco.org/images/0019/001902/190297s.pdf

Organización de las Naciones Unidas para la educación, la Ciencia y la Cultura. (2013). TERCE: Tercer Estudio Regional Comparativo y Explicativo. Recuperado de http://www.unesco.org/new/fileadmin/MULTIMEDIA/FIELD/Santiago/pdf/Primera-

Entrega-TERCE-Final.pdf

Phillips, Beth y Torgesen, Joseph. (2006). Phonemic Awareness and Reading: Beyond the Growth of Initial Reading accuracy. En David Dickinson y Susan Neuman (Eds.), Handbook of Early Literacy Research, (Vol. 2; pp. 101-105). New York: Guilford Publications.

Romero, Rita, Torrado, María y Mesa, Carol. (2006). Emergencia de las capacidades metalingüísticas. Revista Latinoamericana de Psicología, 38(3), 457-475. Recuperado de 05342006000300002

http://pepsic.bvsalud.org/scielo.php?script=sci arttext\&pid=S0120-

Sans, Anna. (2008). ¿Por qué me cuesta tanto aprender? Barcelona: Edebé.

Seymour, Philip. (2007). Modeling Reading: The Dual-Route Approach. En Margaret Snowling y Charles Hulme (Eds.), The Science of reading a Handbook (pp. 9-20). USA: Blackwell Publishing.

Silva, Carmen. (2015). Mejora de la comprensión lectora. España: ladislexia.net

Tapia, Alonso. (2005). Claves para la enseñanza de la comprensión lectora. Revista de Educación, (Extraordinario), 63-93. 